International Journal of Pure and Applied Mathematics

Volume 115 No. 1 2017, 169-186

ISSN: 1311-8080 (printed version); ISSN: 1314-3395 (on-line version)

url: http://www.ijpam.eu

doi: 10.12732/ijpam.v115i1.13

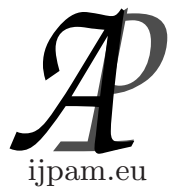

\title{
SHADOWABLE WEAKLY TRANSITIVE SETS ARE HYPERBOLIC
}

\author{
Manseob Lee \\ Department of Mathematics \\ Mokwon University \\ Daejeon, 302-729, KOREA
}

\begin{abstract}
Let $M^{d}(d \geq 2)$ be a closed smooth manifold, and let $f$ be a diffeomorphism on $M$. Let $\Lambda$ be a weakly transitive set for $f$. First, we show that $f$ has the $C^{1}$-stably shadowing property on $\Lambda$ if and only if $\Lambda$ is a hyperbolic basic set. Finally, we prove that for $C^{1}$-generic $f$, a locally maximal weakly transitive set is shadowable if and only if that weakly transitive set is a hyperbolic basic set.
\end{abstract}

AMS Subject Classification: 37C20, 37C25, 37C50, 34D10

Key Words: shadowing, hyperbolic, weakly transitive, transitive set, basic set

\section{Introduction}

In differentiable dynamical systems, robust dynamic property and generic property are a basic tool. For instance, Mañé [12] proved that in two dimensional manifold, if a diffeomorphism is robustly transitive, then it is Anosov. Pugh [14] showed that $C^{1}$ generically, the closure of the set of periodic points is the nonwandering set. Also, Bonatti and Crovisier shown in [3] that $C^{1}$-generically,

$\begin{array}{lr}\text { Received: } & \text { March 3, 2017 } \\ \text { Revised: } & \text { June 28, 2017 } \\ \text { Published: } & \text { June 28, 2017 }\end{array}$

(C) 2017 Academic Publications, Ltd. url: www.acadpubl.eu 
the closure of the set of periodic points is the recurrent set. For generic property, Abdenur and Díaz [1], give a conjecture which is $C^{1}$-generically, a diffeomorphsism has the shadowing property if and only if it is hyperbolic. Actually, the notion of shadowing property often appears in several methods of the modern theory of dynamical system (see [13]). Moreover, the shadowing property usually plays an important role in the investigation of stability theory and ergodic theory.

Let $M=M^{d}(d \geq 2)$ be a closed $C^{\infty}$ manifold, and let $\operatorname{Diff}(M)$ be the space of diffeomorphisms of $M$ endowed with the $C^{1}$-topology. Denote by $d$ the distance on $M$ induced from a Riemannian metric $\|\cdot\|$ on the tangent bundle $T M$. Let $f \in \operatorname{Diff}(M)$.

For $\delta>0$, a sequence of points $\left\{x_{i}\right\}_{i=a}^{b}(-\infty \leq a<b \leq \infty)$ in $M$ is called a $\delta$-pseudo orbit of $f$ if $d\left(f\left(x_{i}\right), x_{i+1}\right)<\delta$ for all $a \leq i \leq b-1$. Let $\Lambda \subset M$ be a closed $f$-invariant set. We say that $\left.f\right|_{\Lambda}$ has the shadowing property if for every $\epsilon>0$ there is $\delta>0$ such that for any $\delta$-pseudo orbit $\left\{x_{i}\right\}_{i=a}^{b} \subset \Lambda$ of $f(-\infty \leq a<b \leq \infty)$, there is a point $y \in M$ such that $d\left(f^{i}(y), x_{i}\right)<\epsilon$ for all $a \leq i \leq b-1$. The point $y$ is called the shadowing point.

Note that $f$ has the shadowing property if and only if $f^{n}$ has the shadowing property for $n \in \mathbb{Z} \backslash\{0\}$.

For given $x, y \in M$, we write $x \rightsquigarrow y$ if for any $\delta>0$, there is a $\delta$-pseudo orbit $\left\{x_{i}\right\}_{i=a}^{b}(a<b)$ of $f$ such that $x_{a}=x$ and $x_{b}=y$. The set of points $\{x \in M: x \leftrightarrow x\}$ is called the chain recurrent set of $f$ and is denoted by $\mathcal{R}(f)$. If we denote the set of periodic points $f$ by $P(f)$, then $P(f) \subset \Omega(f) \subset \mathcal{R}(f)$. Here $\Omega(f)$ is the non-wandering set of $f$. We write $x \rightsquigarrow y$ if $x \rightsquigarrow y$ and $y \rightsquigarrow x$. The relation $\leadsto$ induces on $\mathcal{R}(f)$ an equivalence relation, whose classes are called chain components of $f$. The notion of the weakly transitive set was used in (see $[4,5,18]$ ). We say that $y$ is a weak iterate of $x$ by $f$ if for any neighborhood $U$ of $x$ and $V$ of $y$, there exist $n \geq 1$ and a point $z \in U$ such that $f^{n}(z) \in V$. Let $\Lambda \subset M$ be a closed $f$-invariant set. We say that $\Lambda$ is weakly transitive set if for any neighborhood $W$ of $\Lambda$, and any open sets $U, V$ that intersect $\Lambda$, there exist $n \geq 1$ and a point $z \in U$ such that $f^{n}(z) \in V$ and such that the orbit segment of orbit $\left\{z, f(z), \ldots, f^{n}(z)\right\} \subset W$. Note that by definition, a transitive set is a weakly transitive set. However, the converse is not true. Note that in this paper, we just consider that weakly transitive sets are nontrivial. Here a nontrivial set means that it is not just one orbits. We say that $\Lambda$ is locally maximal if there is a compact neighborhood $U$ of $\Lambda$ such that $\bigcap_{n \in \mathbb{Z}} f^{n}(U)=\Lambda$.

We introduce the notion of the $C^{1}$-stably shadowing property. 
Definition 1.1. Let $\Lambda$ be a closed $f \in \operatorname{Diff}(M)$-invariant set. We say that $f$ has the $C^{1}$-stably shadowing property on $\Lambda$, (or $\Lambda$ is $C^{1}$-stably shadowabe) if there are a compact neighborhood $U$ of $\Lambda$ and a $C^{1}$-neighborhood $\mathcal{U}(f)$ of $f$ such that

(a) $\Lambda=\Lambda_{f}(U)=\bigcap_{n \in \mathbb{Z}} f^{n}(U)$ (locally maximal),

(b) for any $g \in \mathcal{U}(f),\left.g\right|_{\Lambda_{g}(U)}$ has the shadowing property, where $\Lambda_{g}(U)=$ $\bigcap_{n \in \mathbb{Z}} g^{n}(U)$ is the continuation of $\Lambda$.

It is well-known that every $f$ satisfying both Axiom $\mathrm{A}$ and the strong transversality condition has the shadowing property (see [15]). Then, such a system is structurally stable, i.e., there exists $C^{1}$-neighborhood $\mathcal{U}(f)$ of $f$ such that every $g \in \mathcal{U}(f)$ has the shadowing property because $g$ also satisfies Axiom $\mathrm{A}$ and the strong transverslaity condition. In [16], K. Sakai proved that if there is a $C^{1}$-neighborhood $\mathcal{U}(f)$ of $f$ such that for any $g \in \mathcal{U}(f), g$ has the shadowing property then $f$ satisfies both Axiom A and the strong transversality condition. Thus we get the following.

Theorem 1.2. [16] A diffeomorphism $f$ has the $C^{1}$-stably shadowing property if and only if $f$ satisfies both Axiom $A$ and the strong transversality condition.

By the above theorem, the $C^{1}$-interior shadowing property in the whole space is characterized as the structurally stability of the system. Denote by $\mathcal{F}^{*}(M)$ the set of $f \in \operatorname{Diff}(M)$ such that there is a $C^{1}$-neighborhood $\mathcal{U}(f)$ of $f$ such that for any $g \in \mathcal{U}(f)$, every $p \in P(g)$ is hyperbolic. In [8], Hayashi proved that $f \in \mathcal{F}^{*}(M)$ if and only if $f$ satisfies both Axiom A and the nocycle condition. Thus to prove the "only if "part it is enough to the show that if there is a $C^{1}$-neighborhood $\mathcal{U}(f)$ of $f$ such that for any $g \in \mathcal{U}(f), g$ has the shadowing property on $\mathcal{R}(g)$, then $f \in \mathcal{F}^{*}(M)$.

By Hurley [7], the chain recurrent set $\mathcal{R}(f)$ of $f \in \operatorname{Diff}(M)$ is upper semicontinuous, i.e., for any neighborhood $U$ of $\mathcal{R}(f)$, there is $\delta>0$ such that if for $g \in B_{\delta}(f)(g \in \operatorname{Diff}(M))$, then $\mathcal{R}(g) \in U$, where $B_{\delta}(f)$ is a $\delta$-neighborhood of $f$ with $C^{0}$-metric on $\operatorname{Diff}(M)$. Hence, we get the following result of [9].

Theorem 1.3. [9] Let $f \in \operatorname{Diff}(M)$. The chain recurrent set $\mathcal{R}(f)$ of $f$ has the $C^{1}$-stably shadowing property if and only if $f$ satisfies both Axiom $A$ and the no-cycle condition.

Let $\Lambda$ be a closed $f$-invariant set. We say that $\Lambda$ is a basic set if $\Lambda$ is locally maximal and transitive. Then, we know that if $f$ is Axiom A then $\Omega(f)$ consists 
of finitely many basic sets, i.e.,

$$
\Omega(f)=\Lambda_{1} \cup \Lambda_{2} \cup \cdots \cup \Lambda_{n},
$$

where $\Lambda_{i}$ is a basic set, for $i=1, \ldots n$.

We say that $\Lambda$ is hyperbolic if the tangent bundle $T_{\Lambda} M$ has a $D f$-invariant splitting $E^{s} \oplus E^{u}$ and there exists constants $C>0$ and $0<\lambda<1$ such that

$$
\left\|\left.D_{x} f^{n}\right|_{E_{x}^{s}}\right\| \leq C \lambda^{n} \text { and }\left\|\left.D_{x} f^{-n}\right|_{E_{x}^{u}}\right\| \leq C \lambda^{n}
$$

for all $x \in \Lambda$ and $n \geq 0$.

In this paper, we will use the Mañé's techniquics [12]. Then we have

Theorem 1.4. Let $\Lambda$ be a weakly transitive set of $f \in \operatorname{Diff}(M)$. Then $f$ has the $C^{1}$-stably shadowing property on $\Lambda$ if and only if $\Lambda$ is a hyperbolic basic set.

We say that a subset $\mathcal{G} \subset \operatorname{Diff}(M)$ is residual if $\mathcal{G}$ contains the intersection of a countable family of open and dense subsets of $\operatorname{Diff}(M)$. In this case $\mathcal{G}$ is dense in $\operatorname{Diff}(M)$. A property " $\mathrm{P}$ " is said to be $\left(C^{1}\right)$-generic if "P" holds for all diffeomorphisms which belong to some residual subset of $\operatorname{Diff}(M)$. We use the terminology "for $C^{1}$-generic $f$ " to express "there is a residual subset $\mathcal{G} \subset$ $\operatorname{Diff}(M)$ such that for any $f \in \mathcal{G} \ldots$.. Ahn et al[2] proved that $C^{1}$ generically, if a diffeomorphism has the shadowing property on a locally maximal homoclinic class then it is hyperbolic. Recently, Lee and Lee [10] proved that $C^{1}$ generically, if a diffeomorphism $f$ has the shadowing property on homoclinic classes then it is hyperbolic. For the result, we have the following.

Theorem 1.5. For $C^{1}$-generic $f$, a locally maximal weakly transitive set is shadowable if and only if it is a hyperbolic basic set.

\section{Proof of Theorem 1.4}

Let $M$ be as before, and let $f \in \operatorname{Diff}(M)$.

Remark 2.1. Let $\Lambda$ be a nontrivial transitive set. By Pugh's closing lemma [14], there are a sequences diffeomorphisms $\left\{g_{n}\right\}_{n \in \mathbb{N}}$ and a periodic orbit sequence $P_{n}$ of $g_{n}$ with period $\pi\left(P_{n}\right)$ as $n \rightarrow \infty$ such that $g_{n} \rightarrow f$ with $C^{1}$-topology and $\lim P_{n}=\Lambda$. Thus if a transitive set $\Lambda$ is locally maximal in $U$, then there is a periodic point $p \in P(f)$ such that the orbit $\operatorname{Orb}(p) \subset U$. Thus, we know that $p \in \Lambda$. If a transitive set is weakly transitive set, one can see 
that a periodic point belongs to weakly transitive sets. Moreover, a nontrivial transitive set does not contains sinks and sources. Thus a weakly transitive set does not contains sinks and sources.

The following so-called Franks' lemma will play an essential role in our proofs.

Lemma 2.2. [6] Let $\mathcal{U}(f)$ be any given $C^{1}$-neighborhood of $f$. Then there exist $\epsilon>0$ and a $C^{1}$-neighborhood $\mathcal{V}(f) \subset \mathcal{U}(f)$ of $f$ such that for given $g \in \mathcal{V}(f)$, a finite set $\left\{x_{1}, x_{2}, \cdots, x_{k}\right\}$, a neighborhood $U$ of $\left\{x_{1}, x_{2}, \cdots, x_{k}\right\}$ and linear maps $L_{i}: T_{x_{i}} M \rightarrow T_{g\left(x_{i}\right)} M$ satisfying $\left\|L_{i}-D_{x_{i}} g\right\| \leq \epsilon$ for all $1 \leq i \leq k$, there exists $\widetilde{g} \in \mathcal{U}(f)$ such that $\widetilde{g}(x)=g(x)$ if $x \in\left\{x_{1}, x_{2}, \cdots, x_{k}\right\} \cup(M \backslash U)$ and $D_{x_{i}} \widetilde{g}=L_{i}$ for all $1 \leq i \leq k$.

Let $\Lambda$ be a closed $f$-invariant set. If $f$ has the $C^{1}$-stably shadowing property on $\Lambda$ then there are a $C^{1}$-neighborhood $\mathcal{U}(f)$ of $f$ and a compact neighborhood $U$ of $\Lambda$ such that $\bigcap_{n \in \mathbb{Z}} f^{n}(U)=\Lambda$ and for any $g \in \mathcal{U}(f), g$ has the shadowing property on $\Lambda_{g}(U)=\bigcap_{n \in \mathbb{Z}} g^{n}(U)$.

Lemma 2.3. Under the above notation, let $\mathcal{V}(f) \subset \mathcal{U}(f)$ be given by Lemma 2.2. Suppose that $f$ has the $C^{1}$-stably shadowing property on $\Lambda$. Then for any $g \in \mathcal{V}(f)$, every $p \in \Lambda_{g}(U) \cap P(g)$ is hyperbolic.

Proof. See [9, Lemma 3.2]. $\square$

We say that a compact $f$-invariant set $\Lambda$ admits a dominated splitting if the tangent bundle $T_{\Lambda} M$ has a $D f$-invariant splitting $E \oplus F$ and there exists constants $C>0$ and $\lambda \in(0,1)$ such that

$$
\left\|\left.D_{x} f^{n}\right|_{E_{x}}\right\| \cdot\left\|\left.D_{f^{n}(x)} f^{-n}\right|_{F_{f^{n}(x)}}\right\| \leq C \lambda^{n}
$$

for all $x \in \Lambda$ and $n \geq 0$. If $\Lambda$ admits a dominated splitting $T_{\Lambda} M=E \oplus F$ such that $\operatorname{dim} E(x)$ is constant for all $x \in \Lambda$, then there exist a $C^{1}$-neighborhood $\mathcal{U}(f)$ of $f$ and a compact neighborhood $U$ of $\Lambda$ such that for any $g \in \mathcal{U}(f)$, $\left.g\right|_{n \in \mathbb{Z}} g^{n}(U)$ admits a dominated splitting

$$
T_{\bigcap_{n \in \mathbb{Z}} g^{n}(U)} M=E_{g}^{\prime} \oplus F_{g}^{\prime}
$$

with $\operatorname{dim} E_{g}^{\prime}=\operatorname{dim} E$.

Let $\Lambda$ be a closed $f$-invariant set. Suppose that $\left.f\right|_{\Lambda}$ has the $C^{1}$-stably shadowing property; that is, there are a $C^{1}$-neighborhood $\mathcal{U}(f)$ of $f$ and a compact neighborhood $U$ of $\Lambda$ such that for any $g \in \mathcal{U}(f), g$ has the shadowing 
property on $\Lambda_{g}(U)$. Then we will use the Mañé's techniques [12]. Indeed, by Lemma 2.3 the family of periodic sequences of linear isomorphisms of $\mathbb{R}^{\operatorname{dim} M}$ generated by $D g$ ( $g$ is $C^{1}$-near by $f$ ) along the hyperbolic periodic points $q \in$ $\Lambda_{g}(U) \cap P(g)$ is uniformly hyperbolic. That is, there is $\epsilon>0$ such that for any $g$ is $C^{1}$-nearby $f, q \in \Lambda_{g}(U) \cap P(g)$, and any sequence of linear maps $L_{i}: T_{g^{i}(q)} M \rightarrow T_{g^{i+1}(q)} M$ with $\left\|L_{i}-D_{g^{i}(q)} g\right\|<\epsilon(1 \leq i \leq \pi(q)-1), \prod_{i=0}^{\pi(q)-1} L_{i}$ is hyperbolic.

The index of a hyperbolic periodic point $p$ is the dimension of the stable subspace $E^{s}(p)$, denoted by index $(p)=\operatorname{dim} E^{s}(p)$. We have the following result.

Proposition 2.4. [12] Let $\Lambda$ be a weakly transitive set, and let $p \in$ $\Lambda \cap P(f)$ be a hyperbolic point of $f$. Suppose that $f$ has the $C^{1}$-stably shadowing property on $\Lambda$, and let $\mathcal{V}(f)$ be given by Lemma 2.3. Then there are constants $C>0, m>0$ and $0<\lambda<1$ such that

(a) for any $g \in \mathcal{V}(f)$, if $p \in \Lambda_{g}(U) \cap P(g)$ has minimum period $\pi(p) \geq m$, then

$$
\begin{aligned}
& \prod_{i=0}^{k-1}\left\|\left.D_{g^{i m}(p)} g^{m}\right|_{E_{g^{i m}(p)}^{s}}\right\|<C \lambda^{k} \text { and } \\
& \prod_{i=0}^{k-1}\left\|\left.D_{g^{-i m}(p)} g^{-m}\right|_{E_{g^{-i m}(p)}^{u}}\right\|<C \lambda^{k},
\end{aligned}
$$

where $k=[\pi(p) / m]$, and $\Lambda_{g}(U)=\bigcap_{n \in \mathbb{Z}} g^{n}(U)$.

(b) $\Lambda$ admits a dominated splitting $T_{\Lambda} M=E \oplus F$ with $\operatorname{dim} E=\operatorname{index}(p)$.

Lemma 2.5. Let $\Lambda$ be a weakly transitive set of $f$. If $\Lambda$ is locally maximal in $U$ and $f$ has the shadowing property on $\Lambda$, then $\Lambda$ is transitive.

Proof. Let $V_{1}$ and $V_{2}$ be given open sets of $\Lambda$ and fix $x \in V_{1}$ and $y \in V_{2}$. Suppose that $f$ has the shadowing property on a locally maximal $\Lambda$. We can choose $\epsilon>0$ sufficiently small such that $B_{\epsilon}(x) \cap \Lambda \subset V_{1}$ and $B_{\epsilon}(y) \cap \Lambda \subset V_{2}$. Let $0<\delta<\epsilon / 2$ be the number of the shadowing property of $\left.f\right|_{\Lambda}$ for $\epsilon$. Then we take $\delta_{1}>0$ such that $d(a, c)<\delta_{1}$ and $d(b, f(c))<\delta_{1}$ then one can has $d(b, f(a))<\delta$. Take $W=B_{\delta_{1}}(\Lambda)$. At first, we construct $\delta$-pseudo orbit of $f$ as follows. By the definition of weakly transitive set, we can choose $z \in B_{\delta_{1}}(x)$ and $k_{1}>0$ such that $f^{k_{1}}(z) \in B_{\delta_{1}}(y)$ and the orbit segment $\left\{z, f(z), \ldots, f^{k_{1}}(z)\right\} \subset W$. We can take $x_{0}=z$ and $x_{k_{1}}=y$ and $x_{i} \in \Lambda$ such that $d\left(x_{i}, f^{i}(z)\right)<\delta_{1}$ for any $1 \leq i<k_{1}$. Form the choice of $\delta_{1}$ we know that $\left\{x_{i}\right\}_{i=0}^{k_{1}}$ is a $\delta$ pseudo orbit. Similarly, we can choose another $\delta$-pseudo orbit $\left\{x_{i}\right\}_{i=k_{1}}^{k_{1}+k_{2}} \subset \Lambda$ 
such that $x_{k_{1}}=y$ and $x_{k_{1}+k_{2}}=x$. Since $x_{k_{1}+k_{2}}=x_{0}$, we can extend the pseudo orbits $\left\{x_{i}\right\}_{i=0}^{k_{1}+k_{2}}$ periodically to be $\left\{x_{i}\right\}_{i=-\infty}^{\infty}$. By the shadowing property, we have $p \in B_{\epsilon}(x)$ which $\epsilon$-shadows the pseudo orbit $\left\{x_{i}\right\}_{i=-\infty}^{\infty}$. By the local maximal property of $\Lambda$, we have $p \in \Lambda$. By the choice of $\epsilon$ and $k>0$, $p \in B_{\epsilon}(x) \cap \Lambda \subset V_{1}, f^{k_{1}}(p) \in B_{\epsilon}(y) \cap \Lambda \subset V_{2}$. Thus $f^{k_{1}}(p) \in V_{2} \cap f^{k_{1}}\left(V_{1}\right) \neq \emptyset$. Hence $\Lambda$ is transitive.

It is well-known that if $p$ is a hyperbolic periodic point $f$ with period $k$ then the sets

$$
\begin{gathered}
W^{s}(p)=\left\{x \in M: f^{k n}(x) \rightarrow p \text { as } n \rightarrow \infty\right\} \text { and } \\
W^{u}(p)=\left\{x \in M: f^{-k n}(x) \rightarrow p \text { as } n \rightarrow \infty\right\}
\end{gathered}
$$

are $C^{1}$-injectively immersed submanifolds of $M$. A point $x \in W^{s}(p) \cap W^{u}(p)$ is called a homoclinic point of $f$ associated to $p$, and it is said to be a transversal homoclinic point of $f$ if the above intersection is transversal at $x$, i.e., $x \in$ $W^{s}(p) \pitchfork W^{u}(p)$. The closure of the homoclinic points of $f$ associated to $p$ is called the non-transversal homoclinic class of $p$, and it is denoted by $\widetilde{H}_{f}(p)$. The closure of the transversal homoclinic points of $f$ associated to $p$ is called the transversal homoclinic class of $p$, and it is denoted by $H_{f}(p)$. Clearly, $H_{f}(p) \subset$ $\widetilde{H}_{f}(p)$. We consider all periodic points of the saddle type. We say that $p$ and $q$ are homoclinically related, and write $p \sim q$ if $W^{s}(p) \pitchfork W^{u}(q) \neq \emptyset$, and $W^{u}(p) \pitchfork$ $W^{s}(q) \neq \emptyset$, where $\pitchfork$ is transverse intersection. Then we know that if $p \sim q$ then $\operatorname{index}(p)=\operatorname{index}(q)$. Recall that chain transitive sets are characterized as set of all compact invariant sets which are the limit for the Hausdorff metric of a sequence of periodic orbits under the $C^{1}$-generic assumption (see $[4$, Theorem 4])

Let $\left.f\right|_{\Lambda}$ be weakly transitive and $C^{1}$-stably shadowing. Then by Lemma 2.3, we get the following lemma.

Lemma 2.6. Let $\Lambda$ be a weakly transitive set. Suppose that $f$ has the $C^{1}$-stably shadowing property on $\Lambda$. If $p \in \Lambda \cap P(f)$, then $\Lambda \subset H_{f}(p)$.

Proof. Suppose that $f$ has the $C^{1}$-stably shadowing property on $\Lambda$. By Lemma 2.3, for any $p \in \Lambda \cap P(f), p$ is a hyperbolic saddle. Then we can choose $\epsilon_{1}>0$ such that if $d\left(f^{n}(x), f^{n}(p)\right) \leq \epsilon_{1}$ for $n \geq 0$ then $x \in W_{\epsilon_{1}}^{s}(p)$, and if $d\left(f^{n}(x), f^{n}(p)\right) \leq \epsilon_{1}$ for $n \leq 0$ then $x \in W_{\epsilon_{1}}^{u}(p)$. Let $U$ be as in the definition of $C^{1}$-stably shadowing property. To simplify notation in this proof, we may assume that $f(p)=p$. Fix any $0<\epsilon<\epsilon_{1}$, and let $\delta=\delta(\epsilon)>0$ be the number of the shadowing property of $\left.f\right|_{\Lambda}$ for $\epsilon$. By Lemma 2.5, $\Lambda$ is transitive. Since $\Lambda$ is transitive, there is a point $x \in \Lambda$ such that $\omega_{f}(x)=\Lambda$. For any $y \in \Lambda$, we can 
choose $m, k, l \in \mathbb{Z}$ such that $f^{l}(x) \in B_{\delta}(p), f^{k}(x) \in B_{\delta}(y)$, and $f^{m}(x) \in B_{\delta}(p)$. Without loss of generality, we may assume that $m>k>l>0$. Let $k=l+j_{1}$ for some $j_{1}>0$ and $m=k+j_{2}$ for some $j_{2}>0$. Then we can make a finite $\delta$-pseudo orbit as follows:

$$
\begin{aligned}
& \left\{p, f^{l}(x), f^{l+1}(x), \ldots, f^{k-1}(x), f^{k}(x), f^{k+1}(x), \ldots, f^{m-1}(x), p\right\} \\
& =\left\{p, f^{l}(x), f^{l+1}(x), \ldots, f^{l+j_{1}-1}(x), f^{l+j_{1}}(x), f^{l+j_{1}+1}(x), \ldots, f^{l+j_{1}+j_{2}-1}(x), p\right\}
\end{aligned}
$$

Put $x_{-i}=f^{-i}(p)$ for all $i \geq 0, x_{i}=f^{l+i}(x)$ for all $0 \leq i \leq j_{2}-1$, and $x_{j_{1}+j_{2}+i}=f^{m+i}(p)=f^{l+j_{1}+j_{2}+i}(x)$ for all $i \geq 0$. Then

$$
\xi=\left\{\ldots, x_{0}(=p), x_{1}, x_{2}, \ldots, x_{j_{1}}, x_{j_{1}+1}, \ldots, x_{j_{1}+j_{2}}(=p), \ldots\right\}
$$

is a $\delta$-pseudo orbit of $f$. It is clear that $\xi \subset \Lambda$. By the shadowing property, we can choose $z \in B_{\epsilon}(y)$ such that $z \in B_{\epsilon}\left(f^{k}(x)\right)$, and $d\left(f^{i}(z), x_{i}\right)<\epsilon$ for all $i \in \mathbb{Z}$. Therefore, we can find $j>0$ and $j_{1}>0$ such that $d\left(f^{j}(z), p\right)<\epsilon$, and $d\left(f^{-j_{1}}(z), p\right)<\epsilon$. Since $f$ has the shadowing property on $\Lambda$, we see that $d\left(f^{j+i}(z), p\right)<\epsilon$ for $i \geq 0$, and $d\left(f^{-j_{1}-i}(z), p\right)<\epsilon$ for $i \geq 0$. Then $f^{j}(z) \epsilon$ $W_{\epsilon}^{s}(p)$, and $f^{-j_{1}}(z) \in W_{\epsilon}^{u}(p)$. Therefore $z \in f^{-j}\left(W_{\epsilon}^{s}(p)\right) \cap f^{j_{1}}\left(W_{\epsilon}^{u}(p)\right)$. Thus

$$
z \in W^{s}(p) \cap W^{u}(p) \cap B_{\epsilon}(y) \neq \emptyset .
$$

This means that $z \in \widetilde{H}_{f}(p)$. Moreover, $z$ is a transverse intersection point of $W^{s}(p)$ and $W^{u}(p)$ (see [17, Proposition 3.1]). Thus $z \in H_{f}(p)$.

Let $\Lambda$ be a weakly transitive set and locally maximal in $U$. The next lemma says that the periodic points are dense in $\Lambda$ if $f$ has the shadowing property on $\Lambda$.

Lemma 2.7. Let $\Lambda$ be a weakly transitive set. If $f$ has the $C^{1}$-stably shadowing property on $\Lambda$ then $\Lambda=\overline{\Lambda \cap P(f)}$.

Proof. From Lemma 2.6, we know that for any $x \in \Lambda$ and any neighborhood $U$ of $x$, there is a point $y \in U \cap W^{s}(p) \pitchfork W^{u}(p)$. By the locally maximal property of $\Lambda$, we can assume that $\operatorname{Orb}(y) \subset \Lambda$. We know that $\operatorname{Orb}(y) \cup \operatorname{Orb}(p)$ is a hyperbolic set, by the shadowing property of $\operatorname{Orb}(y) \cup \operatorname{Orb}(p)$, there are hyperbolic periodic orbits arbitrarily close to $\operatorname{Orb}(y) \cup \operatorname{Orb}(p)$. By the locally maximal property of $\Lambda$, there are hyperbolic periodic points contained in $\Lambda \cap U$. This ends the proof of lemma.

Let $p$ be a hyperbolic periodic point of $f$, and let $\pi(p)$ is the minimum period of $p$. In [9], the authors proved that if $\Lambda$ is $C^{1}$-stably shadowable then 
it does not have the heterodimensional cycle. Also, they use the result of the Mánẽ, i.e., $\Lambda_{i}(f)=\overline{P_{i}\left(\left.f\right|_{\Lambda}\right)}$ is hyperbolic, where $P_{i}(f)$ is the set of index $i$ of the periodic points. More precisely, denote by $\Lambda_{i}(f)$ the closure of the set of hyperbolic periodic points of $f$ with index $i$. In fact, it is proved in [12] that if there is a $C^{1}$-neighborhood $\mathcal{U}(f)$ of $f$ such that for any $g \in \mathcal{U}(f)$ any periodic points of $g$ is hyperbolic and $\Lambda_{i}(f) \cap \Lambda_{j}(f)=\emptyset$ for $0 \leq i \neq j \leq \operatorname{dim} M$, then $f$ satisfies both Axiom $\mathrm{A}$ and the no-cycle condition. Since the proof is in a neighborhood of $\Lambda_{0}(f) \cup \Lambda_{1}(f) \cup \cdots \cup \Lambda_{n}(f)$, we can see that the result also holds for the definition of $C^{1}$-stably shadowing property on $\Lambda$. Thus, just we show that there are a $C^{1}$-neighborhood $\mathcal{U}(f)$ of $f$ and a compact neighborhood $U$ of $\Lambda$ such that for any $g \in \mathcal{U}(f)$, any periodic point of $\Lambda_{g}(U)$ is hyperbolic and have the same index, where $\Lambda_{g}(U)=\bigcap_{n \in \mathbb{Z}} g^{n}(U)$. This implies there are a $C^{1}$ neighborhood $\mathcal{U}(f)$ of $f$ and a compact neighborhood $U$ of $\Lambda$ such that for any $g \in \mathcal{U}(f), \Lambda_{g}(U)$ is hyperbolic. By Lemma 4.1, we get that for any $\mathcal{U}(f)$, and for any $p, q \in \Lambda_{g}(U) \cap P(g)$ are hyperbolic. In this section, we will show that in fact $p \sim q$.

Proposition 2.8. Let $\Lambda$ be a weakly transitive set. Suppose that $f$ has the $C^{1}$-stably shadowing property on $\Lambda$. Let $\mathcal{U}(f)$ and $U$ be as in the definition. Then for any $g \in \mathcal{U}(f)$, for any $p, q \in \Lambda_{g}(U) \cap P(g)$, we have index $(p)=\operatorname{index}(q)$, where $\Lambda_{g}(U)=\bigcap_{n \in \mathbb{Z}} g^{n}(U)$.

To prove the Proposition 2.8, we need to the following lemma.

Lemma 2.9. Let $\Lambda$ be a weakly transitive set. Suppose that $f$ has the shadowing property on $\Lambda$. Then for any $p, q \in \Lambda \cap P_{h}(f)$,

$$
W^{s}(p) \cap W^{u}(q) \neq \emptyset \text {, and } W^{u}(p) \cap W^{s}(q) \neq \emptyset,
$$

where $P_{h}(f)$ is the set of hyperbolic periodic points of $f$.

Proof. Let $p, q$ be two hyperbolic periodic points of $f$ in $\Lambda$ and let $\epsilon(p)>0$ and $\epsilon(q)>0$ be as before with respect to $p$ and $q$. Suppose that $f$ has the shadowing property on $\Lambda$. Fix $\epsilon=\min \{\epsilon(p), \epsilon(q)\}$. To simplify the notion in the proof, assume that $f(p)=p$ and $f(q)=q$. Let $0<\delta<\epsilon / 2$ be the number of the shadowing property of $f_{\left.\right|_{\Lambda}}$. Then we take $\delta^{\prime}>0$ such that if $d(x, y)<\delta^{\prime}$ and $d\left(x_{1}, f(y)\right)<\delta^{\prime}$ then $d(x, f(y))<\delta$. Take $W=B_{\delta^{\prime}}(\Lambda)$. Let $B_{\epsilon}(p)$ be the neighborhood of $p$, that is, $B_{\epsilon}(p)=\{x \in M: d(x, p)<\epsilon\}$. Since $p, q \in \Lambda$, we can choose $x \in B_{\delta^{\prime}}(p)$ and $k_{1}>0$ such that $f^{k_{1}}(z) \in B_{\delta^{\prime}}(q)$, and the orbit segment $\left\{z, f(z), \ldots, f^{k_{1}}(z)\right\} \subset W$. Then we can take $x_{0}=p$ and $x_{k_{1}}=q$ and $x_{i} \in \Lambda$ such that $d\left(x_{i}, f^{i}(z)\right)<\delta^{\prime}$ for any $1 \leq i<k_{1}$. By the choice of $\delta^{\prime}$, we know that $\left\{x_{i}\right\}_{i=0}^{k_{1}}$ is a $\delta$-pseudo orbit. For the above pseudo orbit, we can 
extend the pseudo orbit $\left\{x_{i}\right\}_{i \in \mathbb{Z}}$ as follows: If $i \leq 0$, then $x_{i}=p$ and if $i \geq k_{1}$ then $x_{i}=q$. Then we get the $\delta$-pseudo orbit

$$
\xi=\left\{\ldots, p, p, x_{1}, x_{2}, \ldots, x_{k_{1}-1}, q, q, \ldots\right\} .
$$

Then clearly, $\xi \subset \Lambda$. Since $f$ has the shadowing property on $\Lambda$, we can choose a point $y \in B_{\epsilon}(p)$ such that

$$
d\left(f^{n}(y), x_{n}\right)<\epsilon,
$$

for $n \in \mathbb{Z}$. Since $x_{-i}=p$ for $i \leq 0$, and $x_{i}=q$ for $i \geq k_{1}$, we know that for $n>0, f^{-n}(y) \in W_{\epsilon}^{u}(p)$ and for $i \geq 0, f^{k_{1}+i}(y) \in W_{\epsilon}^{s}(q)$. Therefore, for $n>0, y \in f^{n}\left(W_{\epsilon}^{u}(p)\right)$ and for $i \geq 0, f^{k_{1}}(y) \in f^{-i}\left(W_{\epsilon}^{s}(q)\right)$. Thus $y \in$ $f^{n}\left(W_{\epsilon}^{u}(p)\right) \cap f^{-k_{1}}\left(f^{-i}\left(W_{\epsilon}^{s}(q)\right)\right) \subset W^{u}(p) \cap W^{s}(q)$, and so,

$$
W^{u}(p) \cap W^{s}(q) \neq \emptyset \text {. }
$$

Let $p \in P(f)$ be a hyperbolic. Then for any $g \in \operatorname{Diff}(M)^{1}$-nearby $f$ there exists a unique hyperbolic periodic point $p_{g} \in P(g)$ nearby $p$ such that $\pi(p)=\pi\left(p_{g}\right)$ and index $(p)=\operatorname{index}\left(p_{g}\right)$. Such a $p_{g}$ is called continuation of $p$.

Proof of Proposition 2.8. Let $\Lambda$ be a weakly transitive set. Suppose $f$ has the $C^{1}$-stably shadowing property on $\Lambda$. Then there are a $C^{1}$-neighborhood $\mathcal{U}_{0}(g) \subset \mathcal{U}(f)$ of $g$ such that for any $g \in \mathcal{U}_{0}(g)$, there are the continuations $p_{g}, q_{g} \in \Lambda_{g}(U)$ and $\left.g\right|_{\Lambda_{g}(U)}$ has the shadowing property. Since $\Lambda=$ $\bigcap_{n \in \mathbb{Z}} f^{n}(U) \subset \operatorname{int} U$, we may assume that $\Lambda_{g}(U) \subset \operatorname{int} U$ for any $g \in \mathcal{U}(f)$ reducing $\mathcal{U}(f)$ if necessary. To derive a contradiction, we may assume that there are hyperbolic $p, q \in \Lambda \cap P(f)$ such that index $(p) \neq \operatorname{index}(q)$. Then $\operatorname{dim} W^{u}(q)+\operatorname{dim} W^{s}(p)<\operatorname{dim} M$ or $\operatorname{dim} W^{s}(q)+\operatorname{dim} W^{u}(p)<\operatorname{dim} M$. We may assume that $\operatorname{dim} W^{s}(p)+\operatorname{dim} W^{u}(q)<\operatorname{dim} M$. Take $x \in W^{s}(p) \cap W^{u}(q)$ and $y \in W^{u}(p) \cap W^{s}(q)$. Clear that $x, y \in \Lambda$. Then as in the proof of $[17$, Proposition 3.2], we can choose $g \in \mathcal{U}(f)$ such that $g$ has a small piece containing $y$ and the orbit $\operatorname{Orb}(y)$ is contained in $U$. Then we can make a $\delta$-pseudo orbit which is not $\epsilon$ shadowed. This is a contradiction.

Let us recall Mañé's ergodic closing lemma in [12]. For any $\epsilon>0$, let $B_{\epsilon}(f, x)$ an $\epsilon$-tubular neighborhood of $f$-orbit of $x$, i.e., $B_{\epsilon}(f, x)=\{y \in M$ : $d\left(f^{n}(x), y\right)<\epsilon$, for some $\left.n \in \mathbb{Z}\right\}$. Let $\Sigma_{f}$ be the set of points $x \in M$ such that for any $C^{1}$-neighborhood $\mathcal{U}(f)$ of $f$ and $\epsilon>0$, there are $g \in \mathcal{U}(f)$ and $y \in P(g)$ satisfying $g=f$ on $M \backslash B_{\epsilon}(f, x)$ and $d\left(f^{i}(x), g^{i}(y)\right) \leq \epsilon$ for $0 \leq i \leq \pi(y)$. 
Remark 2.10. [12, Theorem $A$ ] For any $f$-invariant probability measure $\mu$, we have $\mu\left(\Sigma_{f}\right)=1$.

End of the proof "only if" part of Theorem 1.4. Let $\Lambda$ be a weakly transitive set. Suppose that $f$ has the $C^{1}$-stably shadowing property on $\Lambda$. By Lemma 2.6, $H_{f}(p)=\Lambda_{j}(f)$, where $0<j=\operatorname{index}(p)<\operatorname{dim} M$. Let $\mathcal{U}_{0}(f)$ be the $C^{1}$-neighborhood of $f$ given by Proposition 3.6. To get the conclusion, it is sufficient to show that $\Lambda_{i}(f)$ is hyperbolic, where $\Lambda_{i}(f)=\overline{P_{i}\left(\left.f\right|_{\Lambda}\right)}$, and $i$ is index of $\Lambda$. Fix any neighborhood $U_{i} \subset U$ of $\Lambda_{i}(f)$. Note that by Proposition 2.8, $\Lambda_{j}(f)=\overline{P_{j}\left(\left.f\right|_{\Lambda}\right)}=\emptyset$ if $i \neq j$.

Thus we show the following: let $\mathcal{V}(f) \subset \mathcal{U}_{0}(f)$ be a small connected $C^{1}$ neighborhood of $f$. If any $g \in \mathcal{V}(f)$ with $q=f$ on $M \backslash U_{i}$, then index $(p)=$ index (q) for any $p, q \in \Lambda_{g}(U) \cap P(g)$. Indeed, suppose not, then there are $g_{1} \in \mathcal{V}(f)$ and $q \in \Lambda_{g}(U) \cap P\left(g_{1}\right)$ such that $g_{1}=f$ on $M \backslash U_{i}$ and index $(p) \neq \operatorname{index}(q)$. Suppose that $g_{1}^{n}(q)=q, k=\operatorname{index}(q)$, and define $\gamma: \mathcal{V}(f) \rightarrow \mathbb{Z}$ by

$$
\gamma(g)=\sharp\left\{y \in \Lambda_{g}(U) \cap P(g): g^{n}(y)=y \quad \text { and } \quad \operatorname{index}(y)=k\right\} .
$$

By Lemma 2.3, the function $\gamma$ is continuous, and since $\mathcal{V}(f)$ is connected, it is constant. But the property of $g_{1}$ implies $\gamma\left(g_{1}\right)>\gamma(f)$. This is a contradiction.

We will finished proof of Theorem 1.4 using the proof of Theorem B in [12]. Thus we show that

$$
\liminf _{n \rightarrow \infty}\left\|\left.D_{x} f^{n}\right|_{E_{x}}\right\|=0 \text { and } \liminf _{n \rightarrow \infty}\left\|\left.D_{x} f^{-n}\right|_{F_{x}}\right\|=0
$$

for all $x \in \Lambda$, and thus, the splitting is hyperbolic.

More precisely, we will prove the case of $\liminf _{n \rightarrow \infty}\left\|D_{x} f_{\mid E}^{n}\right\|=0$ (other case is similar). It is enough to show that for any $x \in \Lambda$, there exist $n=n(x)>0$ such that

$$
\prod_{j=0}^{n-1}\left\|\left.D f^{m}\right|_{E_{f m j}(x)}\right\|<1 .
$$

We will derive a contraction. If it is not true, then there is $x \in \Lambda$ such that

$$
\prod_{j=0}^{n-1}\left\|\left.D f^{m}\right|_{E_{f^{m j}(x)}}\right\| \geq 1
$$

for all $n \geq 0$. Thus

$$
\frac{1}{n} \sum_{j=0}^{n-1} \log \left\|\left.D f^{m}\right|_{E_{f^{m j}(x)}}\right\| \geq 0 .
$$


for all $n \geq 0$. Define a probability measure

$$
\mu_{n}:=\frac{1}{n} \sum_{j=0}^{n-1} \delta_{f^{j}(x)}
$$

Then there exists $\mu_{n_{k}}(k \geq 0)$ such that $\mu_{n_{k}} \rightarrow \mu_{0} \in \mathcal{M}_{f}(M)$, as $k \rightarrow \infty$, where $M$ is compact metric space. Thus

$$
\begin{gathered}
\int \log \left\|\left.D f\right|_{E_{x}}\right\| d \mu_{0}=\lim _{k \rightarrow \infty} \int \log \left\|\left.D f\right|_{E_{x}}\right\| d \mu_{n_{k}} \\
=\lim _{k \rightarrow \infty} \frac{1}{n} \sum_{j=0}^{n-1} \log \left\|\left.D f\right|_{E_{f^{j}(x)}}\right\| \geq 0 .
\end{gathered}
$$

By Mañè ([12], page 521),

$$
\int_{\Lambda} \log \left\|\left.D f\right|_{E_{x}}\right\| d \mu_{0}=\int_{\Lambda} \frac{1}{n} \sum_{j=0}^{n-1} \log \left\|\left.D_{f^{j}(r)} f\right|_{E_{f^{j}(x)}}\right\| d \mu_{0} \geq 0
$$

where $\mu_{0}$ is a $f$-invariant measure. Let

$$
B_{\epsilon}(f, x)=\left\{y \in M: d\left(f^{n}(x), y\right)<\epsilon \text { for some } n \in \mathbb{Z}\right\}
$$

and $\Sigma_{f}=\left\{x \in M: d\left(f^{n}(x), y\right)<\epsilon\right.$, there exist $g \in \mathcal{U}(f)$ and $y \in P(g)$ such that $g=f$ on $M \backslash B_{\epsilon}(f, x)$ and $d\left(f^{i}(x), f^{i}(y)\right) \leq \epsilon$ for $\left.0 \leq i \leq \pi(y)\right\}$.

Note that if $x \notin P(f), 0 \leq \pi(y)=N$ such that

$$
d\left(f^{N}(x), f^{N}(y)\right)=d\left(f^{N}(x), y\right) \rightarrow 0
$$

as $N \rightarrow \infty$, then $d(x, y) \rightarrow 0$. So, this is a contradiction.

For any $\mu \in \mathcal{M}_{f}(M), \mu\left(\Sigma_{f}\right)=1$. Then, for any $\mu \in \mathcal{M}_{f}(\Lambda)$,

$$
\mu\left(\Lambda \cap \Sigma_{f}\right)=1
$$

since $\mu(\Lambda)=1$ and $\mu\left(\Sigma_{f}\right)=1$. Thus, $\Lambda=\Lambda \cap \Sigma(f)$ almost everywhere. Therefore,

$$
\int_{\Lambda \cap \Sigma(f)} \lim _{n \rightarrow \infty} \frac{1}{n} \sum_{j=0}^{n-1} \log \left\|\left.D f\right|_{E_{f^{j}(x)}}\right\| d \mu \geq 0 .
$$


By Birkhoff's theorem, and the Ergodic Closing Lemma, we can take $z_{0} \in$ $\Lambda \cap \Sigma(f)$ such that

$$
\lim _{n \rightarrow \infty} \frac{1}{n} \sum_{j=0}^{n-1} \log \left\|\left.D f\right|_{E_{f^{j}\left(z_{0}\right)}}\right\| \geq 0 .
$$

By Proposition 3.6, this is a contradiction. Thus by Proposition 3.6, $z_{0} \notin P(f)$.

Let $K>0, m>0$ and $\lambda \in(0,1)$ be given by Proposition 3.6 and take $\lambda<\lambda_{0}<1$ and $n_{0}>0$ such that

$$
\frac{1}{n} \sum_{j=0}^{n-1} \log \left\|D f_{\left.\right|_{E_{f}{ }^{m}\left(z_{0}\right)} ^{m}}^{m}\right\| \geq \log \lambda_{0}, \quad \text { if } n \geq n_{0} .
$$

Then, by Mañé's ergodic closing lemma we can find $g \in \mathcal{V}_{0}(f) g=f$ on $M \backslash U_{j}$ and $r_{g} \in \Lambda_{g} \cap P(g)$ near by $r$.

Moreover, we know that $\operatorname{index}\left(r_{g}\right)=\operatorname{index}(p)$ since $g=f$ on $M \backslash U_{j}$. Вy applying Lemma 2.2, we can construct $h \in \mathcal{V}_{0}(f)(\subset \mathcal{V}(f)) C^{1}$-nearby $g$ such that

$$
\lambda_{0}^{k} \leq \prod_{i=0}^{k-1}\left\|g_{1}^{i m}\left(r_{g_{1}}\right) h_{\mid E_{g_{1}^{i m}\left(r_{g_{1}}\right)}^{m}}\right\|
$$

(see [12, pages 523-524]). On the other hand, by Proposition 3.6, we see that

$$
\prod_{i=0}^{k-1}\left\|D_{g_{1}^{i m}\left(r_{g_{1}}\right)} h_{\mid E_{g_{1}^{i m}\left(r_{g_{1}}\right)}^{m}}\right\|<K \lambda^{k} .
$$

We can choose the period $\pi\left(r_{g_{1}}\right)$ (>n $n_{0}$ ) of $r_{g_{1}}$ as large as $\lambda_{0}^{k} \geq K \lambda^{k}$. Here $k=\left[\pi\left(r_{g_{1}}\right) / m\right]$. This is a contradiction. Thus,

$$
\liminf _{n \rightarrow \infty}\left\|D_{x} f_{\mid E_{x}}^{n}\right\|=0
$$

for all $x \in \Lambda$. Therefore, $\Lambda$ is hyperbolic. This completes the proof of the "only if" part of Theorem 1.4.

\section{Proof of Theorem $\mathbf{1 . 5}$}

Let $\Lambda$ be a closed $f$-invariant set of $f \in \operatorname{Diff}(M)$ that is locally maximal. In this section, we prove Theorem 1.5 under the additional condition that $C^{1}$ generically, $\Lambda$ is weakly transitive set and $f$ has the shadowing property. To prove Theorem 1.5, we will use Crovisier's result. That is, $C^{1}$-generically, a 
compact $f$-invariant set $\Lambda$ is a weakly transitive set if and only if it is a chain transitive set. Moreover, the chain transitive sets of $C^{1}$-generic diffeomorphisms are approximated in the Hausdorff topology by periodic orbits (see [4]). Again, we use the Mañé's result [12]. At first, we introduce the following that it is well-known Kupka-Smale Theorem.

Lemma 3.1. There is a residual set $\mathcal{G}_{1} \subset \operatorname{Diff}(M)$ such that for any $f \in \mathcal{G}_{1}, f$ has the following properties:

(a) Every periodic orbit is hyperbolic, and

(b) the stable manifolds and the unstable manifolds of the periodic points are transversal.

Lemma 3.2. [4] There is a resudual set $\mathcal{G}_{2} \subset \operatorname{Diff}(M)$ such that for any $f \in \mathcal{G}_{1}, f$ has the following properties:

(a) A chain transitive set of $f$ is the weakly transitive set of $f$.

(b) A compact $f$-invariant set $\Lambda$ is chain transitive if and only if $\Lambda$ is the Hausdorff limit of a sequence of periodic orbits of $f$.

Lemma 3.3. There is a residual set $\mathcal{G}_{3} \subset \operatorname{Diff}(M)$ such that every $f \in \mathcal{G}_{3}$ satisfies the following property: For any closed $f$-invariant set $\Lambda$, if there is a sequence of $f_{n} \in \operatorname{Diff}(M)$ converging to $f$ and a sequence of hyperbolic periodic orbits $\mathcal{O}_{f_{n}}\left(p_{n}\right)$ with index $k$ satisfying $\lim _{n \rightarrow \infty} \mathcal{O}_{f_{n}}\left(p_{n}\right)=\Lambda$, then there is a sequence of hyperbolic periodic orbit $\mathcal{O}_{f}\left(q_{n}\right)$ with index $k$ such that $\lim _{n \rightarrow \infty} \mathcal{O}_{f}\left(q_{n}\right)=\Lambda$.

Proof. See [11, Lemma 2.2]. $\square$

In [12], Mañé' gave a result on the approximation by periodic orbit from measure theoretical viewpoint. We say that a point $x \in M$ is well closable for $f \in \operatorname{Diff}(M)$, if for any $\epsilon>0$ there are $g \in \operatorname{Diff}(M)$ with $d_{1}(f, g)<\epsilon$ such that $d\left(f^{n}(x), g^{n}(p)\right)<\epsilon$ for any $0 \leq n \leq \pi(p)$, where $\pi(p)$ is the period of $p$, and $d_{1}$ is the $C^{1}$-metric. Let $\Sigma_{f}$ denotes the set of well closable points of $f$. In [12], Mañé showed that for any $f$-invariant Borel probability measure $\mu$ on $M, \mu\left(\Sigma_{f}\right)=1$. Let $\mathcal{M}$ be the space of all Borel measures $\mu$ on $M$ with the weak*-topology. Then we know that for any ergodic measure $\mu \in \mathcal{M}$ of $f, \mu$ is supported on a periodic orbit $\mathcal{O}_{f}(p)=\left\{p, f(p), \ldots, f^{\pi(p)-1}(p)\right\}$ if and only if

$$
\mu=\frac{1}{\pi(p)} \sum_{i=0}^{\pi(p)-1} \delta_{f^{i}(p)}
$$


where $\delta_{x}$ is the atomic measure respecting $x$.

Let us mention some involving results. In [4] Crovisier explained that any ergodic invariant probability measure $\mu$ of a $C^{1}$-generic diffeomorphism is the limit of a sequence of invariant measures supported by periodic orbits $\mathcal{O}_{f}\left(p_{n}\right)$. Moreover, the orbits $\mathcal{O}_{f}\left(p_{n}\right)$ converge to the support of $\mu$ in the Hausodrff topology.

Lemma 3.4. There is a residual set $\mathcal{G}_{4} \subset \operatorname{Diff}(M)$ such that for any $f \in \mathcal{G}_{4}, f$ satisfies any ergodic invariant measure $\mu$ of $f$ is the limit of sequence of ergodic invariant measure supported by periodic orbits $\mathcal{O}_{f}\left(p_{n}\right)$ in the weak* topology. Moreover, the orbit $\mathcal{O}_{f}\left(p_{n}\right)$ converges to the support of $\mu$ in the Hausdorff topology.

Proof. See [11, Lemma 2.3].

Denote $P_{h}(f)$ by the set of all hyperbolic periodic points.

Proposition 3.5. There is a residual set $\mathcal{G}^{\prime} \subset \operatorname{Diff}(M)$ such that for any $f \in \mathcal{G}^{\prime}$ if $f$ has the shadowing property on a weakly transitive set $\Lambda$ then for any hyperbolic periodic points $p, q \in \Lambda$,

$$
W^{s}(p) \pitchfork W^{u}(q) \neq \emptyset \quad \text { and } \quad W^{u}(p) \pitchfork W^{s}(q) \neq \emptyset .
$$

Proof. Let $f \in \mathcal{G}^{\prime}=\mathcal{G}_{1}$ and let $p, q \in \Lambda \cap P_{h}(f)$. Suppose that $f$ has the shadowing property on $\Lambda$. By Lemma 2.9 and Lemma 3.1, we know that $C^{1}$ generically, for any hyperbolic $p, q \in \Lambda \cap P(f), W^{s}(p) \pitchfork W^{u}(q) \neq \emptyset$ and $W^{u}(p) \pitchfork W^{s}(q) \neq \emptyset$.

In this section, we show that the following proposition which uses Mañés result. It is a key point to prove Theorem 1.4. We define the residual set $\mathcal{G} \subset \operatorname{Diff}(M)$ as follows: $\mathcal{G}=\mathcal{G}^{\prime} \cap \mathcal{G}_{2} \cap \mathcal{G}_{3} \cap \mathcal{G}_{4}$. Then we have the following proposition.

Proposition 3.6. Let $f \in \mathcal{G}$, and let $\Lambda$ be a weakly transitive set of $f$. If $\Lambda$ is locally maximal and $f$ has the shadowing property on $\Lambda$, then there exist constants $m>0$ and $0<\lambda<1$ such that for any $p \in \Lambda \cap P(f)$,

(a)

$$
\prod_{i=0}^{\pi(p)-1}\left\|\left.D f^{m}\right|_{E^{s}\left(f^{i m}(p)\right)}\right\|<\lambda^{\pi(p)}, \quad \text { and }
$$




$$
\prod_{i=0}^{\pi(p)-1}\left\|\left.D f^{-m}\right|_{E^{u}\left(f^{-i m}(p)\right)}\right\|<\lambda^{\pi(p)} .
$$

(b) $\left\|\left.D f^{m}\right|_{E^{s}(p)}\right\| \cdot \|\left. D f^{-1}\right|_{E^{u}\left(f^{m}(p)\right)}<\lambda^{2}$,

where $\pi(p)$ denotes the period of $p$.

The proof of Proposition 3.6 is in [11]. For convenience, we repeat the proof here.

Proof. Let $f \in \mathcal{G}$, and let $\Lambda$ be a locally maximal weakly transitive set. Then we can choose a $C^{1}$-neighborhood $\mathcal{U}(f)$ of $f$ and a neighborhood $U$ of $\Lambda$ such that every $g \in \mathcal{U}(f)$ has nonhyperbolic periodic orbit which contained in $U$. SUppose that $f$ has the shadowing property on $\Lambda$. The proof is by contradiction. Assume that for any $C^{1}$-neighborhood $\mathcal{U}(f)$ of $f$ and a neighborhood $V \subset U$ of $\Lambda$, we can take $g_{1}, g_{2} \in \mathcal{U}(f)$ and hyperbolic periodic orbits $\mathcal{O}_{g_{1}}\left(q_{1}\right) \subset V$ and $\mathcal{O}_{g_{2}}\left(q_{2}\right) \subset V$ such that index $\mathcal{O}_{g_{1}}\left(q_{1}\right) \neq \operatorname{index} \mathcal{O}_{g_{2}}\left(q_{2}\right)$. Then we can take diffeomorphisms $g_{1, n}, g_{2, n}$ such that $g_{1, n} \rightarrow f, g_{2, n} \rightarrow f$ and two sequences of hyperbolic periodic orbits $\mathcal{O}_{g_{1, n}}\left(q_{1, n}\right), \mathcal{O}_{g_{2, n}}\left(q_{2, n}\right)$ such that

(1) $\mathcal{O}_{g_{1, n}}\left(q_{1, n}\right) \rightarrow \Lambda$, and $\mathcal{O}_{g_{2, n}}\left(q_{2, n}\right) \rightarrow \Lambda$ as $n \rightarrow \infty$,

(2) for $n \in \mathbb{N}$, index $\mathcal{O}_{g_{1, n}}\left(q_{1, n}\right) \neq \operatorname{index} \mathcal{O}_{g_{2, n}}\left(q_{2, n}\right)$.

Then we may assume that

$$
\operatorname{index} \mathcal{O}_{g_{1, n}}\left(q_{1, n}\right)=\operatorname{index} \mathcal{O}_{g_{1, m}}\left(q_{1, m}\right)
$$

and

$$
\operatorname{index} \mathcal{O}_{g_{2, n}}\left(q_{2, n}\right)=\operatorname{index} \mathcal{O}_{g_{2, n}}\left(q_{2, m}\right),
$$

for $n, m \in \mathbb{N}$ by taking a subsequence if necessary. From Lemma 3.4, one can take two sequences of periodic orbits $\mathcal{O}_{f}\left(p_{1, n}\right) \subset V$ and $\mathcal{O}_{f}\left(p_{2, n}\right) \subset V$ such that $\operatorname{index} \mathcal{O}_{f}\left(p_{1, n}\right)=\operatorname{index} \mathcal{O}_{g_{1, n}}\left(q_{1, n}\right)$ and $\operatorname{index} \mathcal{O}_{f}\left(p_{2, n}\right)=\operatorname{index} \mathcal{O}_{g_{2, n}}\left(q_{2, n}\right)$, and

$$
\lim _{n \rightarrow \infty} \mathcal{O}_{f}\left(p_{1, n}\right)=\lim _{n \rightarrow \infty} \mathcal{O}_{f}\left(p_{2, n}\right)=\Lambda,
$$

where $\lim$ is the Hausdorff limit. Since $\Lambda$ is locally maximal in $U$,

$$
\mathcal{O}_{f}\left(p_{1, n}\right), \mathcal{O}_{f}\left(p_{2, n}\right) \subset U
$$

and so $\mathcal{O}_{f}\left(p_{1, n}\right), \mathcal{O}_{f}\left(p_{2, n}\right) \subset \Lambda$. By assumption, $\operatorname{index} \mathcal{O}_{f}\left(p_{1, n}\right) \neq \operatorname{index} \mathcal{O}_{f}\left(p_{2, n}\right)$. However, it is a contradiction by Proposition 3.5. Thus we obtain that all of the indices of periodic orbits of $g \in \mathcal{U}(f)$ are the same. From Mãné's result [12], we take the constants $L>0, m_{1} \in \mathbb{Z}^{+}$and $0<\lambda<1$ such that for any $p \in \Lambda \cap P(f)$ with $\pi(p) \geq L$, 
(a)

$$
\begin{gathered}
\prod_{i=0}^{\pi(p)-1}\left\|\left.D f^{m_{1}}\right|_{E^{s}\left(f^{i m_{1}}(p)\right)}\right\|<\lambda^{\pi(p)}, \quad \text { and }, \\
\prod_{i=0}^{\pi(p)-1}\left\|\left.D f^{-m_{1}}\right|_{E^{u}\left(f^{-i m_{1}}(p)\right)}\right\|<\lambda^{\pi(p)} .
\end{gathered}
$$

(b) $\left\|\left.D f^{m_{1}}\right|_{E^{s}(p)}\right\| \cdot \|\left. D f^{-1}\right|_{E^{u}\left(f^{m_{1}}(p)\right)}<\lambda^{2}$.

Let $\Delta$ be the set of all periodic points in $\Lambda$ whose periods are less than $L$. Since every periodic point of $f$ is hyperbolic, there are only a finite periodic number of periodic point in $\Delta$. Thus $\Delta$ is hyperbolic for $f$. Let $j \in \mathbb{Z}^{+}$such that

$$
\left\|\left.D f^{j m}\right|_{E^{s}(x)}\right\|<\lambda \text { and }\left\|\left.D f^{-j m}\right|_{E^{u}(x)}\right\|<\lambda
$$

for all $x \in \Delta$. Let $m=j m_{1}$. Then this proof is finished.

End of the proof of Theorem1.5. Let $f \in \mathcal{G}$. Suppose that $f$ has the shadowing property on a locally maximal weakly transitive set $\Lambda$. By Proposition 3.5 and Proposition 3.6, we know that $\Lambda$ admits a dominated splitting $T_{\Lambda} M=E \oplus F$ which satisfies $E(p)=E^{s}(p)$ and $F(p)=E^{u}(p)$ for every periodic point $p \in \Lambda$. To show that $D f^{m}$ is contracting on $E$ and $D f^{-m}$ is contracting on $F$.

We now show that $D f^{m}$ is contracting on $E$. Suppose that $D f^{m}$ is not contracting on $E$. Then we can take a point $q \in \Lambda$ such hat

$$
\prod_{i=0}^{k-1}\left\|\left.D f^{m}\right|_{E\left(f^{i m}(q)\right.}\right\| \geq 1
$$

for any $k>0$. Then, as in the proof of [11], we get the contradiction. Thus $D f^{m}$ is contraction on $E$. Similarly we can show that $D f^{-m}$ is contracting on $F . \square$

\section{Acknowledgments}

The author is grateful to X. Wen for many useful and encouraging conversations, and wishes to express his deepest appreciation to the referee for his careful reading of the manuscript, critical comments and valuable suggestions. This work is supported by Basic Science Research Program through the National Research Foundation of Korea (NRF) funded by the Ministry of Science, ICT \& Future Planning (No. 2017R1A2B4001892). 


\section{References}

[1] F. Abdenur and L. J. Díaz, Pseudo-orbit shadowing in the $C^{1}$-topology, Disc. Contin. Dynam. Syst., 2007(2007), 223-245.

[2] J. Ahn, K. Lee and M. Lee, Homoclinic classes with shadowing, J. Inequal. Appl., 2012:97(2012), 1-6.

[3] C. Bonatti and S. Crovisier, Récurrence et généricité, Invent. Math. 158 (2004), 33-104.

[4] S. Crovisier, Peirodic orbits and chain transitive sets of $C^{1}$ diffeomorphisms, Publ. Math. de L'iheś, 104, 87-141.

[5] S. Gan and L. Wen, Heteroclinic cycles and homoclinic closures for generic diffeomorphisms, J. Dynam. Diff. Eq., 15 (2003), 451-471.

[6] J. Franks, Necessary conditions for stability of diffeomorphisms, Trans. Amer. Math. Soc., 158 (1971), 301-308.

[7] M. Hurley, Bifurcations and chain recurrence, Ergodic Th. \& Dynm. Syst., 3(1983), 231-240.

[8] S. Hayashi, Diffeomorphisms in $\mathcal{F}^{1}(M)$ satisfy Axiom A, Ergodic Thoery \& Dynam. Syst., 12 (1992), 233-253.

[9] K. Lee, K. Moriyasu and K. Sakai, $C^{1}$-stable shadowing diffeomorphisms, Discrete Contin. Dynam. Syst., 22 (2008), 683-697.

[10] K. Lee and M. Lee, Shadowable chain recurrence classes for generic diffeomorphisms, Taiwan J. Math., 20(2016),399-409.

[11] K. Lee and X. Wen, Shadowable chain transitive sets of $C^{1}$-generic diffeomorphisms, Bull. Korean Math. Soc. 49(2012), 263-270.

[12] R. Mãné, An ergodic closing lemma, Ann. Math. 116 (1982), 503-540.

[13] S. Y. Pilyugin, "Shadowing in Dynamical Systems.", Lecture Notes in Math. 1706 (Springer Verlag, Berlin, 1999).

[14] C. Pugh, An improved closing lemma and a general density theorem, Amer. J. Math., 89(1967), 1010-1021.

[15] C. Robinson, Stability theorems and hyperbolicity in dynamical systems, Rocky Mountain J. Math., 7(1977), 425-437.

[16] K. Sakai, Pseudo orbit tracing property and strong transversality of diffeomorphisms on closed manifolds, Osaka J. Math. 31 (1994), 373-386.

[17] K. Sakai, $C^{1}$-stably shadowable chain components, Ergodic Theory \& Dynam. Sys., 28 (2008), 987-1029.

[18] L. Wen, Generic diffeomorphisms away from homoclinic tangencies and heterodimensional cycles, Bull. Braz. Math. Soc., 35 (2004), 419-452. 